\title{
Prevalence and risk factors for HIV among people aged 50 years and older in Rombo district, Northern Tanzania
}

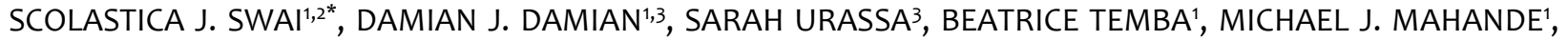 \\ RUNE N. PHILEMON ${ }^{1,3}$ and SIA E. MSUYA ${ }^{1}$ \\ ${ }^{1}$ Kilimanjaro Christian Medical University College, Institute of Public Health, P.O. Box 2240, Moshi, Tanzania \\ ${ }^{2}$ Moshi Municipal Council, P.O. Box 318, Moshi, Tanzania \\ ${ }^{3}$ Kilimanjaro Christian Medical Centre, Moshi, Tanzania
}

\begin{abstract}
Background: It is estimated that over ten percent of the 36.7 million people living with HIV (PLWHIV) globally, are those aged $\geq 50$ years. The objective of this study was to determine the prevalence and risk factors for HIV among adult aged 50 years and above in Rombo district, northern Tanzania.

Methods: This cross sectional study was conducted from April to June 2015 among older adults aged $\geq 50$ year in Rombo district. Interviews were conducted to collect information on socio-demographic characteristics, reproductive and sexual health characteristics of the participants, knowledge on HIV transmission, prevention and risk perception and on awareness and knowledge on voluntary and counselling testing services. In addition, a blood sample was taken for HIV diagnosis.

Results: A total of 600 people were enrolled. Their age ranged from 50 to 90 years with mean of $64.9( \pm$ SD 10.3) years. The HIV prevalence was $1.7 \%(n=10)$ and it was higher among women (2.1\%) than men (1.3\%). Sixtypercent $(n=350)$ were knowledgeable on HIV transmission and only $40 \%(n=216)$ on HIV prevention. In bivariate analysis, age of partner $<50$ years, ever use of condoms, poor knowledge on HIV prevention and perception of risk on HIV were significantly associated with HIV. However, only partner's age of $<50$ years remained significant after controlling for confounders.

Conclusion: HIV prevalence and overall knowledge on its transmission among older adults in this rural population were low. There is a need for strategies to improve HIV preventive knowledge and testing in this group in order to continue keeping the transmission at low levels.

Keywords: HIV, older adults, prevalence, knowledge, risk factors, Tanzania
\end{abstract}

\section{Introduction}

By the end of 2015, there were 36.7 million people living with HIV (PLWHIV) and 2.1 million new infections (UNAIDS, 2016). Sub Saharan Africa (SSA) remains the most affected with 69\% of PLWHIV and $66 \%$ of the new HIV infections (UNAIDS, 2016). The trend of HIV among 15-49 years old individuals has stabilized or declined by $50 \%$ in 26 low and middle income countries. Globally, new HIV infections have declined from 3.2 million in 2000 to 2.1 million in 2015, and in SSA new HIV infections have decreased from 2.4 million in 2001 to 1.8 million in 2015 (UNAIDS, 2014, 2016). In Tanzania, the HIV prevalence has also declined from $7 \%$ in $2003 / 2004$ to $5.1 \%$ in 2011/2012 among people aged $15-49$ years (THMIS 2005,2013).

The trend is different for people aged 50 years or more. Globally, the number of people aged 50 years and above living with HIV has increased; from 2.8 million recorded in 2006 to 3.6 million by the end of the year 2013 (UNAIDS, 2014). Majority (81\%) of the PLWHIV aged $\geq 50$ are from low and middle income countries. SSA has the largest proportion (69\%) of old people living with HIV globally. Eduardo et al, (2014) reported that the number of older adults (50+) who are enrolled in HIV Care and Treatment clinics (CTC) in SSA has increased from $9 \%$ in 2005 to $20 \%$ in

\footnotetext{
* Correspondence E-mail: scolasticajohn@gmail.com
} 
2008. In Tanzania 15\% of the people enrolled in CTC are people aged 50 years or older (Eduardo et al., 2014). Generally, the burden of HIV among older people is expected to increase in SSA and other low and middle income countries due to increased availability/accessibility of antiretroviral therapy (ART) (UNAIDS, 2016). With increased availability of ART, people are living longer and age with HIV (Negin et al., 2012; UNAIDS, 2013, 2016). A number of studies have been done on HIV among people aged 50 years and above in SSA. All these studies have reported a HIV prevalence between 3\% and 14.3\% (Negin et al., 2010, 2012; Nyirenda et al., 2012; Freeman \& Anglewicz, 2012; Kalu et al., 2014). In Tanzania a study in Mufindi and Babati showed a prevalence of $8 \%$ among older adults (Nyigo et al., 2014).

Marked variation has been observed in risk factors for HIV among people aged $\geq 50$ years. In the USA, inconsistent use of condoms was associated with HIV among people older people (Brooks et al., 2012), while in China, transactional sex, inconsistent use of condoms and needlesharing were the risk factors associated with HIV in this age group (Liu et al., 2012). In Zimbabwe, older adults who have never tested for HIV and reported inconsistent condom use were found to have higher HIV prevalence than others (Mahy et al, 2014). In Malawi, multiple partners among men and low level of knowledge on HIV transmission were the risk factors for HIV while in South Africa, low level of knowledge on HIV transmission and prevention were the risk factors for HIV (Negin et al., 2010; Freeman \& Anglewicz, 2012; Nyirenda et al., 2012). In Kenya, conflict within marriage and remarriage after spouse death were the risk factors for HIV among older people (Kyobutungi et al., 2009). Despite increasing burden of HIV among older people, less priority is given to this group in terms of research. There is limited information from population-based studies on predictors and trends of HIV in older adults in Tanzania. This study was therefore carried out to determine the prevalence and risk factors associated with HIV among people aged 50 years and older in a rural district in northern Tanzania.

\section{Materials and Methods}

\section{Study area and design}

This was a cross sectional study conducted from April to June 2015 in Rombo district, northern Tanzania. Rombo district, covering an area of about $1,442 \mathrm{~km}^{2}$ of mainly mountainous area has a population of 260,963 people of which 124,528 are men and 136,435 are women (URT, 2013). Administratively, the district is divided into 5 divisions namely Mengwe, Mkuu, Mashati, Useri and Tarakea, 24 Wards and 68 villages. The largest population of the district depends on agriculture, livestock keeping and small petty business. Few people are employed in the public sector. The district has 43 health facilities; 2 hospitals, 4 health centres and 37 dispensaries.

\section{Study population, sample size and sampling}

The study population was consenting people, aged 50 years and above and who were residents of Rombo district. Multi-stage sampling technique was used to select older people aged 50 years and above. Using Village Registers, a sampling frame of people aged 50 years and above was made and approximately 30 participants were randomly selected from each village. The selected participants were visited at their homes and were informed about the study and were invited to participate. If the participant was not at home as a priori the research team had to visit the house for a minimum 3 times before declaring that the participant could not reached. 


\section{Data collection}

The interviews were conducted at either participant's home or at the village/ ward office depending on the preference of the participant. The questionnaires were administered in Kiswahili and used to collect the required information. Information collected included socio-demographic characteristics, reproductive and sexual health characteristics of the participants, knowledge on HIV transmission, prevention and risk perception and on awareness and knowledge on Voluntary and Counselling Testing services (VCT).

Knowledge on HIV was assessed by asking if the participants had ever heard about HIV. If the response was yes, a participant was supposed to respond to all the other questions on HIV transmission and prevention. The participant was asked to mention any HIV transmission and preventions modes he/she knows. The participant who was able to mention 2 or more ways was considered to have a good knowledge, but participants who mentioned less than 2 methods were categorized to have poor knowledge.

Pretesting counselling for HIV was given to all participants. After the interviews, a finger pricked blood sample was collected for HIV diagnosis using Determine (Alere Medical Co, Ltd, Matsuhidai Matsodoshi Chiba, Japan). Positive results were confirmed using the Unigold (Trinity Biotech plc, Bray, Ireland). All the participants received HIV-post-test counselling. Those who tested positive were referred to Huruma Designated District Hospital for expert management.

\section{Data analysis}

Data were analysed using Statistical Package for Social Sciences version 22. Descriptive statistics were used to summarize data. Categorical data were summarized in percentages. Continuous variables were summarized by using mean or median with their respective measures of dispersion. Odds ratio with their $95 \%$ confidence interval were used to measure the strength of association between HIV and some of socio-demographic and behavioural risk factors. To get independent predictors of HIV, logistic regression analysis was conducted and any variable with P-value of $<0.1$ in the bivariate analysis were entered in the model. P-value of $<0.05$ was taken as a statistical significant result.

\section{Ethical consideration}

The study received ethical approval from Ethical Committee of Kilimanjaro Christian Medical University College (Certificate number 795). Permission to conduct study was also sought from the Rombo District Authority. Information meetings were held with all wards/villages leaders of selected sites to inform them about the study, its objectives and benefits. Before enrolment, the researchers explained the purpose of the study to participants and those who agreed to participate, gave a written consent. Participants who were unable to write, right thumb was regarded as a signature. To maintain confidentiality only numbers were used in the questionnaires and laboratory forms.

\section{Results}

\section{Socio-demographic characteristics}

A total of 600 individuals participated in the study. The age of the participants ranged from $50-$ 90 years with mean age of 64.9 ( \pm SD 10.3) years. Of the 600 participants, $52 \%$ were males; $68 \%$ had primary education; $69 \%$ were married or cohabiting. Economically, majority of the participants (88.2\%) had an income of less than USD 30 per month; $3.5 \%$ were receiving pension; $7.2 \%$ were currently employed (Table 1 ). 
Table 1: Association between socio-demographic characteristics and HIV $(\mathrm{N}=600)$

\begin{tabular}{|c|c|c|c|c|c|}
\hline Variable & Response & No. & HIV+ N (\%)* & COR $(95 \% \mathrm{Cl})$ & P-value \\
\hline \multirow[t]{2}{*}{ Sex } & Male & $311(51.8)$ & $4(1.3)$ & 1 & \\
\hline & Female & $289(48.2)$ & $6(2.1)$ & $1.63(0.46-5.83)$ & 0.533 \\
\hline \multirow[t]{3}{*}{ Age group (years) } & $50-59$ & $214(35.7)$ & $6(2.8)$ & $6.0(0.73-50.99)$ & \\
\hline & $60-69$ & $174(29.0)$ & $3(1.7)$ & $3.7(0.38-35.91)$ & \\
\hline & $\geq 70$ & $212(35 \cdot 3)$ & $1(0.5)$ & 1 & 0.06 \\
\hline Education & None/Primary & $558(93.0)$ & $8(1.4)$ & 1 & \\
\hline Marital status & Single/Widow & $185(30.8)$ & $3(1.6)$ & $0.9(0.25-3.76)$ & 0.990 \\
\hline \multirow[t]{2}{*}{ Partner's age (years)** } & $\geq 50$ & $361(74 \cdot 5)$ & $3(0.8)$ & 1 & \\
\hline & $<50$ & $66(15.5)$ & $4(6.1)$ & $7.69(1.68-35.24)$ & 0.013 \\
\hline \multirow[t]{2}{*}{ Monthly Income (US\$) } & $<30$ & $529(88.2)$ & $9(1.7)$ & 1 & \\
\hline & $\geq 30$ & $71(11.8)$ & $1(1.4)$ & $0.83(0.10-6.61)$ & 0.98 \\
\hline In pension scheme & Yes & $21(3 \cdot 5)$ & $1(4.8)$ & $1.24(0.13-11.66)$ & 0.852 \\
\hline \multirow[t]{2}{*}{ Health insurance } & No & $250(41.7)$ & $2(0.8)$ & 1 & \\
\hline & Yes & $350(58.3)$ & $8(2.3)$ & $2.90(0.61-0.206)$ & 0.206 \\
\hline
\end{tabular}

Key: *= Row percentage; **=173 participants did not have partners; COR= Crude Odds Ratio

\section{Prevalence of HIV and risk factors}

The prevalence of HIV among participants was $1.7 \%$ (10/600). The prevalence was slightly higher among women $2.1 \%(n=6)$ than men $1.3 \%(n=4)$. However, the difference was not statistically significant (OR 1.63 [ $95 \% \mathrm{Cl}: 0.46$ - 5.83]). The prevalence of HIV was $2.8 \%$ among participants aged 50-59 years old, $1.7 \%$ among those aged $60-69$ years and $0.5 \%$ in participants aged 70 years and above.

Table 2: Association between sexual behaviour and HIV characteristics $(\mathrm{N}=600)$

\begin{tabular}{|c|c|c|c|c|c|}
\hline Variable & Response & No (\%) & HIV+ N(\%)* & $\operatorname{COR}(95 \% \mathrm{Cl})$ & P-value \\
\hline \multirow[t]{2}{*}{ No. Children $(\mathrm{N}=588)$} & $<4$ Children & $82(13.9)$ & $2(2.4)$ & 1 & \\
\hline & $\geq 4$ Children & $506(86.1)$ & $8(1.6)$ & $0.64(0.13-3.08)$ & 0.637 \\
\hline \multirow[t]{2}{*}{ Sex in past 3 month } & No & $290(48.3)$ & $7(2.4)$ & 1 & \\
\hline & Yes & $310(51.7)$ & $3(1.0)$ & $0.39(0.10-1.54)$ & 0.210 \\
\hline \multirow{2}{*}{$\begin{array}{l}\text { No. sexual partners past } 12 \\
\text { months }\end{array}$} & No partner & $195(32.5)$ & $3(1.5)$ & 1 & \\
\hline & $\geq 1$ Partner & $405(67.5)$ & $7(1.7)$ & $1.13(0.29-4.40)$ & 0.990 \\
\hline \multirow{2}{*}{$\begin{array}{l}\text { Lifetime number of sexual } \\
\text { partners }\end{array}$} & 1 partner & $275(45.8)$ & $7(2.5)$ & 1 & \\
\hline & $2+$ partners & $325(54.2)$ & $3(0.9)$ & $0.36(0.09-1.39)$ & 0.199 \\
\hline \multirow[t]{2}{*}{ Transactional sex } & No & $520(86.7)$ & $9(1.7)$ & & \\
\hline & Yes & $80(13.3)$ & $1(1.3)$ & $0.72(0.09-5.75)$ & 0.990 \\
\hline \multirow[t]{2}{*}{ Ever use of condoms } & No & $518(86.3)$ & $5(1.0)$ & 1 & \\
\hline & Yes & $82(13.7)$ & $5(6.1)$ & $6.66(1.89-23.55)$ & 0.006 \\
\hline
\end{tabular}

Key: * row percentage

People with partner's aged less than 50 years had 8-times higher odds of being HIV-positive compared to those whose partners were 50 years and older $(p$-value $=0.013$ ). Age, education 
level, marital status, alcohol intake, income level, employment and economic dependency were not associated with HIV, (Table 1).

About $52 \%$ and $68 \%$ of the 600 participants reported to be sexual active in the past 3 and 12 months respectively. Thirteen percent reported to have ever used a condom (Table 2). Report of ever use of condoms was significantly associated with HIV ( $p$ - value 0.006) (Table 2).

Table 3: Association between HIV knowledge on HIV, prevention and VCT use and HIV infection ( $\mathrm{N}=583$ )

\begin{tabular}{|c|c|c|c|c|c|}
\hline Variable & Response & No (\%) & HIV+ N(\%)* & COR $(95 \% \mathrm{Cl})$ & P-value \\
\hline \multirow[t]{2}{*}{ Knowledge on transmission } & Poor & $233(40.0)$ & $4(1.1)$ & 1 & \\
\hline & Good & $350(60.0)$ & $6(2.5)$ & $2.29(0.64-8.19)$ & 0.192 \\
\hline \multirow[t]{2}{*}{ Knowledge of prevention } & Poor & $367(63.0)$ & $8(2.2)$ & $2.44(1.04-14.88)$ & \\
\hline & Good & $216(37.0)$ & $2(0.9)$ & 1 & 0.004 \\
\hline \multirow[t]{2}{*}{ Risk perception for HIV } & No risk & $426(73.1)$ & $4(0.9)$ & 1 & \\
\hline & At risk & $157(26.9)$ & $6(3.8)$ & $4.19(1.17-15.06)$ & 0.027 \\
\hline \multirow[t]{2}{*}{ Ever used VCT services } & No & $241(41.3)$ & $2(0.8)$ & 1 & \\
\hline & Yes & $342(58.7)$ & $8(2.3)$ & $2.86(0.60-13.59)$ & 0.208 \\
\hline
\end{tabular}

Key: * row percentage

Majority of participants (97.2\%) have heard about HIV, and $86 \%$ knew the places to get Voluntary Counselling and Testing services (VCT) for HIV. However only $37 \%$ of the 600 participants had good knowledge on HIV prevention, $60 \%$ had good knowledge on HIV transmission, and 59\% reported to have ever tested for HIV (Table 3). Participants with poor knowledge on HIV prevention had significantly 2.4 times higher odds of being HIV positive than those with good HIV prevention knowledge. People who perceived that they were at risk for HIV had significantly higher HIV prevalence $(3.8 \%)$ than those who perceived they were not at risk $(0.9 \%) ; \mathrm{p}-$ value 0.027 (Table 3).

\section{Independent predictors of HIV}

Logistic regression was done for all the factors associated with HIV at $p<10 \%$ level in the bi-variate analysis. Only partners age of less than 50 years remained significantly associated with HIV Adjusted Odds Ratio 5.21 (95\% Cl: 1.04 - 26.02) (Table 4).

Table 4: Logistic regression for factors associated with HIV

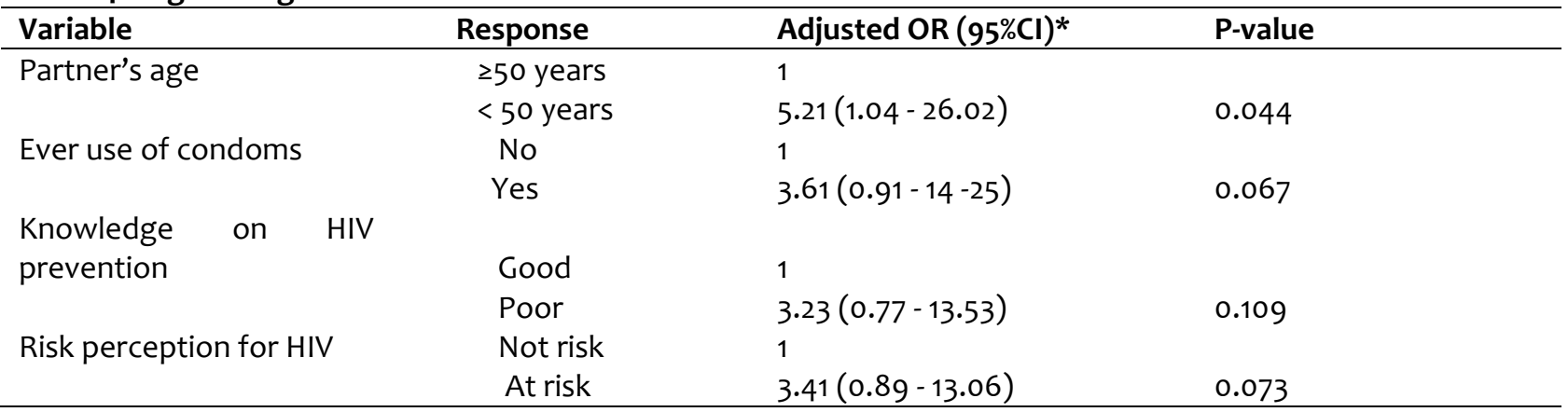

**Adjusted for age groups, partners age, employment in the past, ever use of condoms, knowledge on HIV prevention and risk perception for HIV 


\section{Discussion}

The prevalence of HIV among older people was low and declined with increasing age. It was lower compared to that among people aged 50 years or older elsewhere in Africa (Freeman \& Anglewicz, 2012; Kalu et al., 2014) and Tanzania (Nyigo et al., 2014). A previous a population survey in Tanzania has also reported low HIV prevalence in the general population in this rural setting (THMIS, 2013). However, the fact that older people with HIV are diagnosed late, start ART care with advanced disease or lower CD4 and end with mortality thus they are not reflected in a population based could also be another explanation for the low prevalence among older people (Muteyedzi et al., 2011; Negin et al., 2012). Some studies have also shown that older age on its own is a risk factor for mortality, and HIV positive people aged 50 or older have 3-4 times higher mortality than their younger counterparts (Negin et el., 2010; Eduardo et al., 2014).

In Tanzania, though the HIV prevalence among women aged $15-49$ years in general population is significantly higher among women (6.2\%) than men (3.8\%), the findings of our current study indicate similar prevalence between men and women aged 50 years or more. Contrary to our findings, studies in Malawi and Nigeria have reported a significant difference in HIV prevalence between women and men aged 50 years or older (Freeman \& Anglewicz, 2012; Kalu et al., 2014).

In general knowledge on HIV transmission and prevention methods was poor in this rural population of people aged 50 years or older. About two-thirds of the participants had poor knowledge on HIV preventive methods. Lower knowledge on HIV prevention among older people compared to younger ones has also been reported in South African (Negin \& Cumming, 2010) and Kenya (Kyobutungi et al., 2009). In this study older people with poor knowledge on HIV prevention had higher odds of being positive than their counterparts with good knowledge. This points/ brings attention to the gap in the way preventive efforts are designed. Most communitybased educational interventions in Sub-Saharan Africa, including Tanzania target people aged 15 49 years, adolescents, or pregnant population (Negin \& Cumming, 2010; Negin et al, 2012; UNAIDS, 2013). Strategies to improve general HIV knowledge among the older groups are needed.

Many of the participants were sexually active during and in the past one year prior to the study. Similar findings have been observed in Nigeria and Malawi (Freeman \& Anglewicz, 2012; Kalu et al., 2014). This is contrary to a wide belief in Africa that, older people are not sexually active and hence they are not at risk for HIV (Kyobutungi et al., 2009; UNAIDS, 2013). In this study, people aged 50 or older who assessed themselves to be at higher risk for HIV had 4 times higher odds of being HIV positive. Thus programmes that target to increase the number of people who knows their HIV status to $90 \%$ by 2020, should also target this group (UNAIDS, 2016). Report of ever use of condoms in this study was associated with 6 times higher odds of being HIV positive than others. Condom use may be a marker of higher risk behaviour in this population as previously reported elsewhere in Tanzania (Msuya et al., 2006; Mmbaga et al., 2007). Or it may be that people who know they are HIV positive are more likely to use condoms due to counselling received during testing.

Some of the limitations of the study include the fact, been cross sectional nature, it is difficult to know the temporal nature of observed associations. Participants could also have recall problem on some issues explored. Moreover, the information on HIV prevalence and associated risk factors was collected from a rural setting of one region and may not be generalizable for all other regions or urban settings. Despite the limitations, this was a population based study thus providing baseline information on HIV prevalence, sexual behaviour characteristics and level of 
HIV knowledge among older people in Rombo District. Use of laboratory method to diagnose HIV adds validity of the findings, contrary to other studies that relied on reported information to give HIV prevalence.

We conclude that HIV prevalence among people aged 50 years and older in this rural population is low; and that the overall knowledge on HIV and transmission is low. We recommend strengthening information, education and communication interventions especially targeting older people to improve their knowledge and skills in HIV prevention. Further studies on HIV risk and sexual behaviour in urban settings are needed, to provide a picture of HIV epidemic in older adults in the country. The need to diagnose HIV-positive older people earlier cannot be overemphasized due to the fact they have higher disease progression, higher mortality and less clinical response compared than younger people.

\section{Competing interests}

The authors declare that they have no competing interests.

\section{Authors' contribution}

SEM, DJD, RNP designed the study; SJ, DJD, SU, BT, SEM contributed to data collection. DJD, SEM, MJM analysed the data. MJM, DJD, SEM interpreted the results. All the authors read and approved the final version of the manuscript.

\section{Acknowledgements}

This study was funded by the Mentored Research Program in collaboration with HRSA-funded KCMC MEPI Grant Number T84HA21123-02 of the US National Institutes of Health. We thank the participants for their time and willingness to give information. We also thank the District Executive Director and District Medical Officer of Rombo for permission to conduct the study in the district. We are grateful to the Wards and Village leaders for introducing the research team in their respective areas.

\section{References}

Eduardo, E., Lamb, M.R., Kandula, S., Howard, A., Mugisha, V., Kimanga, D., Kilama, B., El-Sadr, E. \& Elul, B. (2014) Characteristics and outcomes among older HIV-positive adults enrolled in HIV programs in four Sub-Saharan African countries. PLoS One 9(7): e 103864.

Freeman, E. \& Anglewicz, P. (2012) HIV prevalence and sexual behaviour at older ages in rural Malawi. International Journal of STD \& AIDS 23: 490-496.

Liu, H., Lin, X., Xu, Y., Chen, S., Shi, J. \& Morisky, D. (2012) Emerging HIV epidemic among older adults in Nanning China. AIDS Patient Care and STDs 26: 565-567.

Kalu, O.S., Ngozi, O., Prince, U.E., Fiona, R., Bernard, O.O., George, O.C., Simeon, A.N., Arther, E.A. \& Anthony, O.I. (2014) HIV Infection among 50 Years and above Attending Counselling and Testing (HCT) in Nnewi, Nigeria. World Journal of AIDS 4, 93-98.

Kyobutungi, C., Ezeh, A.C., Zulu, E. \& Falkingham, J. (2009) HIV/AIDS and the health of older people in the slums of Nairobi, Kenya: results from a cross sectional survey. BMC Public Health 9:153. 
Mahy, M., Autenrieth, C.S., Stanecki, K. \& Wynd, S. (2014) Increasing trends in HIV prevalence among people aged 50 years and older: evidence from estimates and survey data. AIDS 28 (Suppl 4): S453-S459.

Mmbaga, E., Hussain, A., Leyna, G.H., Mnyika, K.S., Sam, N.E. \& Klepp, K-I. (2007) Prevalence and risk factors for HIV-1 infection in rural Kilimanjaro region of Tanzania: implications for prevention and treatment. BMC Public Health 7:58.

Msuya, S.E., Mbizvo, E., Hussain, A., Uriyo, J., Sam, N.E. \& Pedersen, S.B. (2006) HIV among pregnancy women in Moshi Tanzania: the role of sexual behaviour, male partner characteristics and sexual transmitted infections. AIDS Research and Therapy 3:27.

Mutevedzi, P.C., Lessells, R.J., Rodger, A.J. \& Newell, M-L. (2011) Association of age with mortality and virology and immunological response to antiretroviral therapy in rural South African adults. PLoS One 6: e21795.

Negin, J. \& Cumming, R.G. (2010) HIV infection in older adults in sub-Saharan Africa: extrapolating prevalence from existing data. Bulletin of the World Health Organization 88: 847-853.

Negin, J., Wariero, J., Cumming, R.G., Mutuo, P. \& Pronyk, P.M. (2010) High rates of AIDS-related mortality among older adults in rural Kenya. Journal of Acquired Immune Deficiency Syndrome 55:239-244.

Negin, J., Barnighausen, T., Lundgren, J.D. \& Mills, E.J. (2012) Aging with HIV in Africa: the challenges of living longer. AIDS 26(Supply1): S1-S5.

Nyigo, V., Kilale, A., Kilima, S., Shayo, E.H., Senkoro, K.P., Mshana, J., Mushi, A.K., Matemba, L. \& Massaga, J. (2014). Magnitude of HIV infection among older people in Mufindi and Babati districts of the Tanzania mainland. HIV/AIDS Research and Palliative Care 6: 75-79.

Nyirenda, M., Chatterji, S., Falkingham, J., Mutevedzi, P., Hosegood, V. \& Kowa, P. (2012) An investigation of factors associated with the health and well-being of HIV-infected or HIV affected older people in rural South Africa. BMC Public Health 12:259.

THMIS (2005) HIV/AIDS and Malaria Indicators Survey 2003-04. ORC Macro and Tanzania Bureau of Statistics, Dar es Salaam, Tanzania.

THMIS (2013) HIV/AIDS and Malaria Indicators Survey 2011- 1012. ORC Macro and Tanzania Bureau of Statistics, Dar es Salaam, Tanzania.

UNAIDS (2013) Global AIDS Epidemic Update 2012. United Nations Programme on HIV/AIDS, Geneva, Switzerland: (Available from: http://www.unaids.org/en/).

UNAIDS (2014) The Gap Report. Global AIDS Response Progress Reporting 2014.United Nations Programme on HIV/AIDS; 2014 Geneva, Switzerland: (Available from: http://www.unaids.org/en/).

UNAIDS (2016) AIDS by the numbers: AIDS is not over but it can be. United Nations Programme on HIV/AIDS; 2016 Geneva, Switzerland. (Available from: http://www.unaids.org/en/).

URT (2013) 2012 Population and Housing Census: population distribution by administrative areas. National Bureau of Statistics and Office of the Chief Government Statistics, Dar es Salaam, 2013. 